\title{
Thermoelectric transport of mesoscopic conductors coupled to voltage and thermal probes
}

\author{
David Sánchez and Llorenç Serra \\ Institut de Física Interdisciplinària i de Sistemes Complexos IFISC (CSIC-UIB), E-07122 Palma de Mallorca, Spain and \\ Departament de Física, Universitat de les Illes Balears, E-07122 Palma de Mallorca, Spain
}

(Received 18 July 2011; revised manuscript received 10 October 2011; published 14 November 2011)

\begin{abstract}
We investigate the basic properties of the thermopower (Seebeck coefficient) of phase-coherent conductors under the influence of dephasing and inelastic processes. Transport across the system is caused by a voltage bias or a thermal gradient applied between two terminals. Inelastic scattering is modeled with the aid of an additional probe acting as an ideal potentiometer and thermometer. We find that inelastic scattering reduces the conductor's thermopower and, more unexpectedly, generates a magnetic field asymmetry in the Seebeck coefficient. The latter effect is shown to be a higher-order effect in the Sommerfeld expansion. We discuss our result by using two illustrative examples. First, we consider a generic mesoscopic system described within random matrix theory and demonstrate that thermopower fluctuations disappear quickly as the number of probe modes increases. Second, the asymmetry is explicitly calculated in the quantum limit of a ballistic microjunction. We find that asymmetric scattering strongly enhances the effect and discuss its dependence on temperature and Fermi energy.
\end{abstract}

DOI: 10.1103/PhysRevB.84.201307

PACS number(s): 73.23.-b, 73.50.Lw, 73.63.Kv, 05.70.Ln

Introduction. Recent advances in heat measurements have enabled to envisage promising thermoelectric applications at the mesoscale. ${ }^{1}$ In particular, there have been a number of proposals ranging from thermal analogs of electronic rectifiers ${ }^{2}$ and transistors ${ }^{3}$ to efficient converters of heat into electricity ${ }^{4}$ which can be shown to reach optimized configurations. ${ }^{5}$ Of fundamental importance is the experimental verification of the carriers' charge sign using thermopower techniques only. ${ }^{6}$

Since these devices operate at the quantum regime of transport, it is of primary interest to investigate dephasing effects which may be detrimental to their performance. Additionally, energy flow at finite temperature is expected to be particularly sensitive to inelastic transitions inside the sample due, e.g., to coupling to phonons. This problem has been addressed only very recently. 7,8

A convenient way to introduce dephasing and energy relaxation in a mesoscopic conductor is based on the voltage probe model. ${ }^{9}$ A fictitious terminal is attached to the sample such that the net current flowing through the probe is set to zero. Hence, a carrier absorbed by the probe is reemitted into the conductor with an unrelated phase. The clear advantage of this approach lies in its simplicity and its independence on the microscopic details of the phase-randomizing processes. Therefore, universal properties of generic conductors can be investigated this way. ${ }^{10,11}$ The model has been recently applied to find the temperature and chemical potential profiles of an array of quantum dots. ${ }^{12}$

Including a third terminal acting as a dephasing probe requires to extend the scattering approach to the multiterminal case. For thermoelectric transport, this was achieved by Butcher. ${ }^{13}$ Experimentally, there are already available data for paradigmatic mesoscopic systems such as two-terminal point contacts ${ }^{14}$ and chaotic cavities. ${ }^{15}$ The multiterminal case, however, has been less explored. A very recent exception is Ref. 16, where a voltage drop is generated transversally to a horizontal thermal gradient in a four-terminal setup with an asymmetric scatterer.
Here we investigate incoherent scattering effects on the thermopower (Seebeck coefficient $S$ ), defined at linear response as the ratio of voltage $\Delta V$ to temperature $\Delta \theta$ differences applied between two terminals (1 and 2 in Fig. 1) at vanishing current $I$,

$$
\left.S \equiv \frac{\Delta V}{\Delta \theta}\right|_{I=0},
$$

in the presence of an attached probe (terminal 3 in Fig. 1) acting as an ideal potentiometer and thermometer. Quite generally, we find that $S$ decreases as compared to the case without the probe and, strikingly, the presence of incoherent scattering due to the probe causes the development of a magnetic field asymmetry in $S$. To illustrate our findings, we analyze (i) a mesoscopic conductor with generic properties (a chaotic cavity) and (ii) a ballistic wire in the quantum limit (a few propagating modes) with an asymmetric scatterer. In the latter case, we explicitly calculate the size of the magnetoasymmetry. This result is relevant in view of recent predictions that relate this asymmetry to the efficiency limits of thermal devices in converting a temperature gradient into electrical work. ${ }^{17,18}$

Model. We consider a mesoscopic conductor coupled to three terminals. Thermoelectric transport is described by the electric current $I_{\alpha}$ and the heat current $Q_{\alpha}$ flowing from terminal $\alpha=1,2,3$. In the linear response regime, the transport equations read

$$
\begin{aligned}
I_{\alpha} & =\sum_{\beta} G_{\alpha \beta} V_{\beta}+\sum_{\beta} L_{\alpha \beta} \theta_{\beta}, \\
Q_{\alpha} & =\sum_{\beta} M_{\alpha \beta} V_{\beta}+\sum_{\beta} K_{\alpha \beta} \theta_{\beta},
\end{aligned}
$$

where $e V_{\alpha}=E_{F}-\mu_{\alpha}$ are voltage shifts away from the common Fermi energy $E_{F}$, with $\mu_{\alpha}$ the electrochemical potential of lead $\alpha$, while $\theta_{\alpha}=\theta-T_{\alpha}$ measures deviations of the reservoir temperature $T_{\alpha}$ from the common (bath) 
(a)

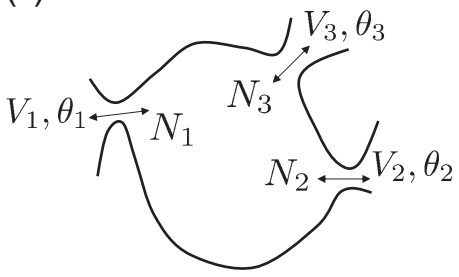

(b)

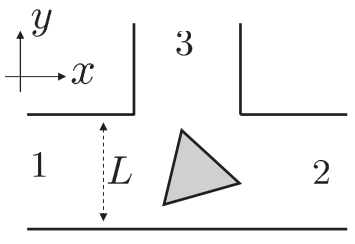

FIG. 1. Sketches of (a) a chaotic cavity attached to voltage and thermal reservoirs and (b) a microjunction with an asymmetric scatterer coupled to a probe as a model of a quantum wire with incoherent scattering.

temperature $\theta$. The transport coefficients are expressed in terms of the transmission probability $T_{\alpha \beta}$ :

$$
\begin{gathered}
G_{\alpha \beta}=-g_{V} \int d E\left(N_{\alpha} \delta_{\alpha \beta}-T_{\alpha \beta}\right) f_{0}^{\prime}, \\
L_{\alpha \beta}=-\frac{g_{V}}{e \theta} \int d E\left(E-E_{F}\right)\left(N_{\alpha} \delta_{\alpha \beta}-T_{\alpha \beta}\right) f_{0}^{\prime}, \\
M_{\alpha \beta}=-\theta L_{\alpha \beta}, \\
K_{\alpha \beta}=\frac{g_{V}}{e^{2} \theta} \int d E\left(E-E_{F}\right)^{2}\left(N_{\alpha} \delta_{\alpha \beta}-T_{\alpha \beta}\right) f_{0}^{\prime},
\end{gathered}
$$

with $g_{V}=2 e^{2} / h$ the conductance quantum, $N_{\alpha}$ the number of propagating channels in lead $\alpha$, and $f_{0}^{\prime}$ the energy derivative of the Fermi distribution function evaluated at $V_{\alpha}=\theta_{\alpha}=0$. In Eq. (6) we have used the Onsager reciprocity relations between cross terms.

Isothermal case. We first consider the case where the temperature probe is an externally fixed parameter. This would correspond, e.g., to a phonon subsystem maintained at a different temperature. ${ }^{8}$ The probe thus works as an ideal voltmeter with voltage $V_{3}$ determined from the condition $I_{3}=0$,

$$
\begin{aligned}
V_{3}= & \frac{G_{31} V_{1}+G_{32} V_{2}}{G_{31}+G_{32}}+\frac{L_{31}}{G_{31}+G_{32}}\left(\theta_{1}-\theta_{3}\right) \\
& +\frac{L_{32}}{G_{31}+G_{32}}\left(\theta_{2}-\theta_{3}\right) .
\end{aligned}
$$

We substitute Eq. (8) into the expression for $I_{1}$, from which we find the current $I=I_{1}=-I_{2}$ flowing through the system:

$$
\begin{aligned}
-I= & g V_{12}+L_{12} \theta_{12}+L_{13} \theta_{13} \\
& -\frac{G_{13}}{G_{31}+G_{32}}\left[L_{31} \theta_{13}+L_{32} \theta_{23}\right] .
\end{aligned}
$$

$I$ is a function of voltage $V_{i j}=V_{i}-V_{j}(i, j=\{1,2,3\})$ and temperature $\theta_{i j}=\theta_{i}-\theta_{j}$ differences, as should be. In Eq. (9), $-g=G_{12}+G_{13} G_{32} /\left(G_{31}+G_{32}\right)$ is the well-known expression for the conductance in the presence of a voltage probe in the purely electric case for which all temperature shifts are set to zero. ${ }^{9}$

In the isothermal configuration, the probe is maintained at the same temperature as the common bath $\left(\theta_{3}=0\right)$. We are then free to specify the precise form of the temperature gradient $\theta_{1}-\theta_{2} \neq 0$. We choose the symmetric bias $\theta_{1}=-\theta_{2}=\Delta \theta / 2$, which is commonly employed in actual measurements. ${ }^{16}$ We then compute the thermopower from Eq. (1) for $V_{1}-V_{2}=\Delta V$ :

$$
S=\frac{1}{g}\left[L_{12}+\frac{1}{2} L_{13}+\frac{1}{2} \frac{G_{13}}{G_{31}+G_{32}}\left(L_{32}-L_{31}\right)\right] .
$$

Equation (10) shows two contributions to the thermopower as compared to the case without phase randomization for which $S=-L_{12} / G_{12}$. The first part corresponds to direct inelastic scattering with the probe and is proportional to the term $L_{13}$, as can be expected from an analogy with the purely electric case (cf., $g$ above). The second part is more surprising - it is nonzero only if there is an asymmetry between the transmissions into the probe of those carriers injected from lead 1 and lead $2\left(L_{32} \neq L_{31}\right)$. This term has no counterpart in the purely electric case. A similar asymmetry effect, but with phonon carriers, has been recently shown to be crucial in the development of rectification effects in dielectric junctions. ${ }^{19}$

Adiabatic case. More interesting is the case when the probe plays simultaneously the role of an ideal potentiometer and thermometer. Then, not only the charge current but also the energy flux is zero at the probe. These two conditions determine $V_{3}$ and $\theta_{3} .{ }^{20}$ Recently, it has been suggested that electronic local temperatures can be consistently defined introducing thermal probes. ${ }^{21}$

Imposing $I_{3}=0$ and $Q_{3}=0$, we find

$$
\begin{gathered}
V_{32}=-\frac{\left(G_{31} K_{33}+\theta L_{31} L_{33}\right) V_{12}+\left(L_{31} K_{33}-K_{31} L_{33}\right) \theta_{12}}{G_{33} K_{33}+\theta L_{33}^{2}}, \\
\theta_{32}=-\frac{\left(G_{33} K_{31}+\theta L_{31} L_{33}\right) \theta_{12}+\left(G_{31} L_{33}-L_{31} G_{33}\right) \theta V_{12}}{G_{33} K_{33}+\theta L_{33}^{2}},
\end{gathered}
$$

which we substitute in the equation $I_{1}=0$ in order to obtain the thermopower $S=\Delta V / \Delta \theta=V_{12} / \theta_{12}$,

$$
\begin{aligned}
S= & {\left[G_{13}\left(L_{31} K_{33}-K_{31} L_{33}\right)+G_{33}\left(L_{13} K_{31}-L_{11} K_{33}\right)\right.} \\
& \left.+\theta L_{33}\left(L_{13} L_{31}-L_{11} L_{33}\right)\right] /\left[\theta L_{33}\left(G_{11} L_{33}-G_{31} L_{13}\right)\right. \\
& \left.+G_{33}\left(\theta L_{13} L_{31}+G_{11} K_{33}\right)-G_{13}\left(G_{31} K_{33}+\theta L_{31} L_{33}\right)\right] .
\end{aligned}
$$

Chaotic cavity. We now consider a generic mesoscopic sample-a metallic quantum dot whose classical analog displays chaotic dynamics [see Fig. 1(a)]. Its isotropic properties permits us to treat the cavity as an effectively zero-dimensional object characterized with a mean level spacing $\delta$. Transport occurs when the cavity is coupled to external reservoirs usually via quantum point contacts with a large number $\left(N_{1}\right.$ and $\left.N_{2}\right)$ of propagating channels. The experimentally relevant case deals with clean samples. ${ }^{22}$ As a consequence, transport is ballistic and its corresponding statistics is well described by random matrix theory (RMT). ${ }^{23}$ In what follows, we assume $e V_{\alpha}, k_{B} \theta_{\alpha}<E_{T}$, with $E_{T}=\left(N_{1}+N_{2}\right) \delta$ the Thouless energy.

Using a Sommerfeld expansion, one finds approximate expressions for the response coefficients in Eqs. (4)-(7) in terms of the transmission functions $T_{\alpha \beta}$ and their energy derivatives $T_{\alpha \beta}^{\prime}$ evaluated at the Fermi level: $G_{\alpha \beta}=g_{V}\left(N_{\alpha} \delta_{\alpha \beta}-T_{\alpha \beta}\right)$, $L_{\alpha \beta}=g_{\theta} T_{\alpha \beta}^{\prime}$, and $K_{\alpha \beta}=-g_{\theta}\left(N_{\alpha} \delta_{\alpha \beta}-T_{\alpha \beta}\right) / e$, where $g_{\theta}=$ $(2 e / h)\left(\pi^{2} k_{B}^{2} \theta / 3\right)$. Note that the ratio $\theta g_{V} / e g_{\theta}=\pi^{2} k_{B}^{2} / 3$ 
depends on universal constants only, representing the fundamental quantum of thermal conductance of a perfectly transmitting mode with averaged reservoir temperature $\theta .^{24}$

We investigate the statistical properties of the thermopower, in particular, its mean value and variance. In the limit $N_{\alpha} \gg 1$ we can calculate correlations of $T_{\alpha \beta}$ and $T_{\alpha \beta}^{\prime}$ within RMT. We consider the cases $\beta=1$ (orthogonal ensemble) and $\beta=2$ (unitary ensemble) corresponding, respectively, to the presence and the absence of time-reversal symmetry. We define the functions $A_{\mu \nu}=N_{\mu} N_{\nu} / N_{t}^{2}-\delta_{\mu \nu} N_{\mu} / N_{t}$ and $B_{\rho \sigma}=\sqrt{2}\left(N_{\rho} N_{\sigma} / N_{t}^{3}-\delta_{\rho \sigma} N_{\rho} / N_{t}^{2}\right)$, where $\mu, \nu, \rho, \sigma$ are lead indices and $N_{t}$ is the total number of modes. Then, using Ref. 25, we derive the useful relations

$$
\begin{gathered}
\left\langle T_{\mu \nu}\right\rangle=\delta_{\mu \nu} N_{\mu}-N_{\mu} N_{\nu} / N_{t}+\delta_{\beta 1} A_{\mu \nu}, \\
\left\langle T_{\mu \nu}^{\prime}\right\rangle=0 \\
\left\langle T_{\mu \nu} T_{\rho \sigma}\right\rangle=A_{\mu \rho} A_{\nu \sigma}+\delta_{\beta 1} A_{\mu \sigma} A_{\nu \rho}, \\
\left\langle T_{\mu \nu}^{\prime} T_{\rho \sigma}^{\prime}\right\rangle=B_{\mu \rho} B_{\nu \sigma}+\delta_{\beta 1} B_{\mu \sigma} B_{\nu \rho} .
\end{gathered}
$$

From Eq. (15) we immediately see that the mean vanishes, $\langle S\rangle=0$. This result was found in Ref. 26 for the case without dephasing. The reason is clear: The $L$ coefficients are functions of the energy derivative of the scattering matrix, but this is zero on average (the derivative can be positive or negative for a specific sample of the ensemble but fluctuates on average at around zero). The fluctuations are described by $\operatorname{var} S=$ $\left\langle S^{2}\right\rangle-\langle S\rangle^{2}$, which are generally nonzero. For definiteness, we set $N_{1}=N_{2}=N$. Then, the thermopower fluctuations for both the isothermal and adiabatic cases are governed by the same expression:

$$
\operatorname{var} S=\frac{8 \pi^{6} k_{B}^{4} \theta^{2}}{9 e^{2} \delta^{2}} \frac{1+\delta_{\beta 1}}{\left(2 N+N_{3}\right)^{4}} .
$$

Our result generalizes the thermopower fluctuations to the case of finite dephasing. Note that the fluctuations are not universal and vanish quickly as the mode number increases. Strikingly, the same functional dependence $\left(\mathrm{N}^{-4}\right)$ appears in the magnetoasymmetry of the weakly nonlinear conductance ${ }^{27}$ since it also depends on the energy derivative of the transmission. The fluctuations are twice larger in the absence of magnetic field. Importantly, incoherent scattering effects reduce drastically the fluctuations of $S$ since these have a quantum origin and the probe introduces decoherence in the system. To leading order, one has ( $\left.\operatorname{var} S-\operatorname{var} S^{N_{3}=0}\right) / \operatorname{var} S^{N_{3}=0}=1-$ $2 N_{3} / N+O\left(N_{3}\right)^{2}$.

In the adiabatic case, we have neglected in $S$ terms that do not contribute to the variance (e.g., terms that involve three $L$ 's are of higher order). Then, Eq. (13) can be approximated as

$$
S \simeq \frac{1}{g}\left[L_{12}+\frac{G_{32} L_{13}}{G_{31}+G_{32}}+\frac{G_{13}\left(G_{31} L_{32}-G_{32} L_{31}\right)}{\left(G_{31}+G_{32}\right)^{2}}\right] .
$$

Since for symmetric couplings $\left(N_{1}=N_{2}=N\right)$ the prefactors of $L_{\alpha \beta}$ in Eq. (19) are, on the ensemble average, equal to those of the isothermal case [Eq. (10)], we obtain the same expression for var $S$. Thus, only asymmetric couplings $\left(N_{1} \neq\right.$ $N_{2}$ ) could distinguish between the two cases.
Magnetic field asymmetry. In a two-terminal conductor, the linear conductance is an even function of the applied magnetic field $B$. This fundamental symmetry remains unchanged even after elimination of the probe coupled to a mesoscopic conductor since $g(B)=g(-B)$. This can be seen by recasting $g$ as $g=G_{11}-G_{13} G_{31} / G_{33}$, which is manifestly symmetric under $B$ reversal. In contrast, while the two-terminal thermopower $S=-L_{11} / G_{11}$ is manifestly an even function of $B$, this statement does not hold in the presence of a probe in the adiabatic case. ${ }^{18}$ From Eq. (13) we find the thermopower magnetoasymmetry $\Phi=S(B)-S(-B)$ :

$$
\begin{aligned}
\Phi= & {\left[G_{33}\left(L_{13} K_{31}-L_{31} K_{13}\right)+L_{33}\left(G_{31} K_{13}-G_{13} K_{31}\right)\right.} \\
& \left.+K_{33}\left(G_{13} L_{31}-G_{31} L_{13}\right)\right] /\left[\theta L_{33}\left(G_{11} L_{33}-G_{31} L_{13}\right)\right. \\
& +G_{33}\left(\theta L_{13} L_{31}+G_{11} K_{33}\right) \\
& \left.-G_{13}\left(G_{31} K_{33}+\theta L_{31} L_{33}\right)\right],
\end{aligned}
$$

which is generally nonzero. Then, incoherent asymmetry changes the symmetry of the Seebeck coefficient.

We recall that the leading order in a Sommerfeld expansion reads $K_{\alpha \beta} \simeq-\left(\pi^{2} k_{B}^{2} \theta / 3 e^{2}\right) G_{\alpha \beta}$. Substituting in Eq. (20), one finds $\Phi=0$. As a consequence, $B$ asymmetries in the thermopower are a higher-order effect. This implies that the size of $\Phi$ will decrease quickly as temperature decreases [typically, as $\theta^{3}$ (Ref. 28)]. The leading-order Sommerfeld approximation neglects terms of order $\left(k_{B} \theta / E_{F}\right)^{4}{ }^{29}$ This factor is rather small in macroscopic metals and it is thus not necessary to consider higher orders. But in low-dimensional systems the $B$ asymmetry of $S$ should be visible since $E_{F}$ and $\theta$ can be of the same order. We note in passing

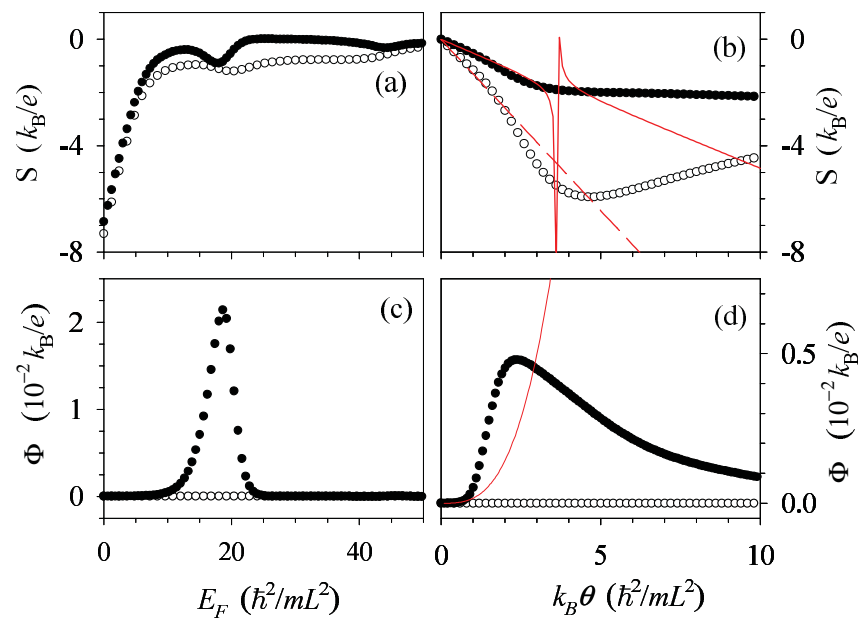

FIG. 2. (Color online) Numerical results for the thermopower (a),(b) and thermopower $B$ asymmetry (c),(d) of a microjunction with an asymmetric scatterer [Fig. 1(b)] as a function of Fermi energy (a),(c) and temperature (b),(d). Solid (open) symbols correspond to the presence (absence) of the voltage and thermal probe. Solid (dashed) lines in (b) are obtained using the Sommerfeld expansion to lowest order with (without) the probe. Note the strong deviation at moderately low temperatures. Solid line in (d) shows the $\Phi \propto \theta^{3}$ dependence of the $B$ asymmetry at low temperature. Parameters: Magnetic length $\hbar c / e B=L^{2}$, temperature $k_{B} \theta=\hbar^{2} / m L^{2}$ [(a),(c)], and Fermi energy $E_{F}=10 \hbar^{2} / m L^{2}[(\mathrm{~b}),(\mathrm{d})]$. 
that the conductance $g$ is, in contrast, always $B$ symmetric, independently of the Sommerfeld approximation.

Numerical simulations. Clearly, for a chaotic cavity the thermopower magnetoasymmetry vanishes on average. Moreover, we expect the fluctuations to be exceedingly small since $\Phi$ depends, to leading order in $\theta$, on the second derivative of the transmission. Therefore, it is more natural to estimate the size of $\Phi$ in a different mesoscopic system-a microjunction as in Fig. 1(b). Thermopowers yielding $0.6 \mu \mathrm{V} / \mathrm{K}$ have been recently detected in a similar setup. ${ }^{16}$ The asymmetric scatterer deflects the electronic trajectories differently depending on the $B$ direction. Therefore, we expect an asymmetry in $S$ when $B$ is inverted.

Our prediction is confirmed by the numerical calculations of Fig. 2. We compute $T_{\alpha \beta}(E)$ using a grid discretization of the Schrödinger equation and, subsequently, the response coefficients from Eqs. (4)-(7) with Gauss-Legendre quadratures. ${ }^{28}$ The upper panels show that adding the voltage and thermal probe reduces the thermopower absolute value as a function of both $E_{F}$ and $\theta$, in agreement with our previous results. In Fig. 2(b) we show that $S$ deviates from the lowest-order Sommerfeld expansion as temperature increases both with and without the probe. We emphasize that the thermopower is $B$ asymmetric only if the probe is present, as shown in
Figs. 2(c) and 2(d). In Fig. 2(d) we plot the asymmetry $\Phi$ as a function of temperature and show that $\Phi$ increases as $\theta^{3}$ at low temperature. The maximum value of $\Phi$ depends on the details of the scatterer and the system's parameters. We find that $\Phi$ can reach values as high as $2 \%$ for Fermi energies close to the activation of the second mode $E_{F} \simeq 19.7 \hbar^{2} / m L^{2}$.

Conclusions. To summarize, we have investigated incoherent scattering effects on the thermoelectric transport of a mesoscopic conductor using a fictitious probe acting as an ideal potentiometer and thermometer. Our main findings are as follows: (i) a general expression for the quantum fluctuations of the Seebeck coefficient upon elimination of the probe valid for both isothermal and adiabatic probes, and (ii) a magnetic field asymmetry of the thermopower, requiring both inelastic and dephasing processes, which becomes apparent only when higher-order terms are considered in a Sommerfeld expansion. Odd-in- $B$ thermopowers in Andreev interferometers have been experimentally observed ${ }^{30}$ and theoretically studied. ${ }^{31}$ We hope that our results will motivate the experimental detection of asymmetric thermopowers in normal systems.

Acknowledgments. We thank M. Büttiker and R. López for fruitful discussions. This work has been supported by the MICINN Grant No. FIS2008-00781.
${ }^{1}$ F. Giazotto, T. T. Heikkilä, A. Luukanen, A. M. Savin, and J. P. Pekola, Rev. Mod. Phys. 78, 217 (2006).

${ }^{2}$ M. Terraneo, M. Peyrard, and G. Casati, Phys. Rev. Lett. 88, 094302 (2002).

${ }^{3}$ L. Wang and B. Li, Phys. Rev. Lett. 99, 177208 (2007).

${ }^{4}$ L. E. Bell, Science 321, 1457 (2008).

${ }^{5}$ R. Sánchez and M. Büttiker, Phys. Rev. B 83, 085428 (2011).

${ }^{6}$ P. Reddy, S-Y. Jang, R. A. Segalman, and A. Majumdar, Science 315, 1568 (2007).

${ }^{7}$ M. Leijnse, M. R. Wegewijs, and K. Flensberg, Phys. Rev. B 82, 045412 (2010).

${ }^{8}$ O. Entin-Wohlman, Y. Imry, and A. Aharony, Phys. Rev. B 82, 115314 (2010)

${ }^{9}$ M. Büttiker, Phys. Rev. B 33, 3020 (1986); IBM J. Res. Dev. 32, 63 (1988).

${ }^{10}$ H. U. Baranger and P. A. Mello, Phys. Rev. B 51, 4703 (1995).

${ }^{11}$ P. W. Brouwer and C. W. J. Beenakker, Phys. Rev. B 55, 4695 (1997).

${ }^{12}$ P. A. Jacquet, J. Stat. Phys. 134, 709 (2009).

${ }^{13}$ P. N. Butcher, J. Phys. Condens. Matter 2, 4869 (1990).

${ }^{14}$ L. W. Molenkamp, Th. Gravier, H. van Houten, O. J. A. Buijk, M. A. A. Mabesoone, and C. T. Foxon, Phys. Rev. Lett. 68, 3765 (1992).

${ }^{15}$ S. F. Godijn, S. Möller, H. Buhmann, L. W. Molenkamp, and S. A. van Langen, Phys. Rev. Lett. 82, 2927 (1999); A. S. Dzurak, C. G. Smith, C. H. W. Barnes, M. Pepper, L. Martín-Moreno, C. T. Liang, D. A. Ritchie, and G. A. C. Jones, Phys. Rev. B 55, 10197(R) (1997).

${ }^{16}$ J. Matthews, D. Sánchez, M. Larsson, and H. Linke, e-print arXiv:1107.3179.
${ }^{17}$ G. Benenti, K. Saito, and G. Casati, Phys. Rev. Lett. 106, 230602 (2011).

${ }^{18}$ K. Saito, G. Benenti, G. Casati, and T. Prosen, Phys. Rev. B 84, 201306 (2011)

${ }^{19}$ Y. Ming, Z. X. Wang, Z. J. Ding, and H. M. Li, New J. Phys. 12, 103041 (2010).

${ }^{20}$ H.-L. Engquist and P. W. Anderson, Phys. Rev. B 24, 1151 (1981)

${ }^{21}$ A. Caso, L. Arrachea, and G. S. Lozano, Phys. Rev. B 81, 041301(R) (2010).

${ }^{22}$ A. G. Huibers, S. R. Patel, C. M. Marcus, P. W. Brouwer, C. I. Duruöz, and J. S. Harris Jr., Phys. Rev. Lett. 81, 1917 (1998).

${ }^{23}$ H. U. Baranger and P. A. Mello, Phys. Rev. Lett. 73, 142 (1994); R. A. Jalabert, J.-L. Pichard, and C. W. J. Beenakker, Europhys. Lett. 27, 255 (1994).

${ }^{24}$ K. Schwab, E. A. Henriksen, J. M. Worlock, and M. L. Roukes, Nature (London) 404, 974 (2000).

${ }^{25}$ M. L. Polianski and P. W. Brouwer, J. Phys. A 36, 3215 (2003).

${ }^{26}$ S. A. van Langen, P. G. Silvestrov, and C. W. J. Beenakker, Superlattices Microstruct. 23, 691 (1998).

${ }^{27}$ D. Sánchez and M. Büttiker, Phys. Rev. Lett. 93, 106802 (2004); Int. J. Quantum Chem. 105, 906 (2005).

${ }^{28}$ See Supplemental Material at http://link.aps.org/supplemental/ 10.1103/PhysRevB.84.201307 for details on the low temperature dependence of the thermopower asymmetry and on our numerical simulations.

${ }^{29}$ N. W. Ashcroft and N. D. Mermin, Solid State Physics (Saunders College, Philadelphia, PA, 1976), p. 761.

${ }^{30} \mathrm{P}$. Cadden-Zimansky, J. Wei, and V. Chandrasekhar, Nat. Phys. 5, 393 (2009).

${ }^{31} \mathrm{Ph}$. Jacquod and R. S. Whitney, Europhys. Lett. 91, 67009 (2010). 